\title{
A Rational Approach Towards a New Ferrocenyl Pyrrolidine for Stereoselective Enamine Catalysis
}

\author{
Diego Petruzziello, ${ }^{[a]}$ Marco Stenta, ${ }^{[b]}$ Andrea Mazzanti, ${ }^{[c]}$ and Pier Giorgio Cozzi*[a]
}

Research in the field of organocatalysis has grown exponentially in the last decade, ${ }^{[1]}$ and organocatalytic modes of activation are currently employed in the synthesis of complex natural products ${ }^{[2]}$ and drugs. ${ }^{[3]}$ Most of the stereoselective organocatalytic reactions are based on the two basic activation modes ${ }^{[4]}$ of enamino ${ }^{[5]}$ and iminum catalysis, ${ }^{[6]}$ as displayed by selected secondary amines bearing a pyrrolidine core. ${ }^{[7]}$

The nickname "work-horses"[8] of enantioselective organocatalysis was assigned to proline, proline derivatives, or other five-membered heterocycles (structures I-V, Figure 1) due to their extensive use in this field. In particular the most commonly used diaryl-pyrrolidine derivatives, the Hayashi and Jørgensen organocatalysts, ${ }^{[9]}$ are silylated at the

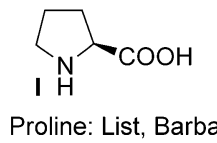

Proline: List, Barbas

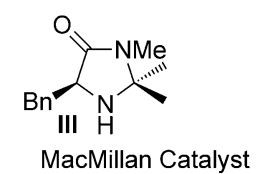

MacMillan Catalyst

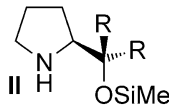

$\mathrm{R}=\mathrm{Ph}:$ Hayashi catalyst $\mathrm{R}=3,5-\mathrm{CF}_{3} \mathrm{Ph}$ : Jørgensen catalyst $\mathrm{R}=n \mathrm{C}_{6} \mathrm{H}_{14} ;$ Palomo catalyst

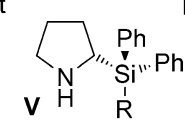

$\mathrm{R}=\mathrm{Me}$, Bolm and Strohmann catalyst $\mathrm{R}=t \mathrm{Bu}$, Franz catalyst

Figure 1 . The catalysts extensively used as "work horses" in organocatalysis.

[a] Dr. D. Petruzziello, Prof. P. G. Cozzi

ALMA MATER STUDIORUM

Dipartimento di Chimica "G. Ciamician"

Via Selmi 2, 40126 Bologna (Italy)

Fax: (+39) 051-2099456

E-mail: piergiorgio.cozzi@unibo.ir

[b] Dr. M. Stenta

Institute of Bioengineering, School of Life Science

Ecole Polythechnique Fédérale de Lausanne

EPFL, Lausanne, 1015 (Switzerland)

[c] Prof. A. Mazzanti

ALMA MATER STUDIORUM, Dipartimento di Chimica Industriale "Toso Montanari" Viale Risorgimento 40136, Bologna (Italy)

Supporting information for this article is available on the WWW under http://dx.doi.org/10.1002/chem.201300959.

oxygen atom (catalyst II), which allows for additional structural diversification by varying the groups on the silicon atom. In this case, the presence of the aryl groups is required for stereo direction. On the other hand, Palomo and co-workers ${ }^{[10]}$ have demonstrated the use of a pyrrolidine bearing a long alkyl chain for reactions carried out in the presence of water. Maruoka has shown that a 2-tritylpyrrolidine is also a quite effective organocatalyst, ${ }^{[11]}$ and more recently, Bolm, Christmann, and Strohmann, and Franz have independently synthetized new organocatalysts based on silylated pyrrolidines. ${ }^{[12]}$ In most of the examples cited, the presence of a diaryl or triaryl moiety is required to fix the conformation of the enamine, and to effectively shield one face from the attack of electrophiles.

The "geminal-diaryl effect" (stereodirection through conformational fixation) is a well-known result of bulky substituents and it has been exploited throughout the field of stereoselective organic synthesis. ${ }^{[13]}$ Not only are two aryl groups, like phenyl, capable of fixing the conformation around a neighboring single bond, but they also become powerful stereodirectors in chiral molecules, in which the two aryl groups become diastereotopic, as in BINAP $\left(2,2^{\prime}\right.$ bis(diphenylphosphino)-1,1'-binaphthyl $)^{[14]}$ or in TADDOL ( $\alpha, \alpha, \alpha, \alpha$-tetraaryl-1,3-dioxolane-4,5-dimethanols). ${ }^{[15]}$ Even in the modified Evans auxiliary DIOZ (3-(1-methylethyl)-5,5diphenyloxazolidin-2-one) the diastereotopic aryl groups improve functional-group stereoselectivity. ${ }^{[16]}$

In this communication, we present the synthesis of new, effective ferrocenyl pyrrolidine catalysts bearing alkyl chains. In this catalyst the preferred conformation of the pyrrolidine ring is imposed by the interaction between a ferrocene moiety and the two alkyl groups. In addition, we proved that the enamine formed by these new organocatalysts is effective in benchmark organocatalytic reactions.

Ferrocene can be considered a "privileged framework" for the construction of effective chiral ligands. ${ }^{[17]}$ Surprisingly, pyrrolidine bearing a ferrocene moiety has not been considered as a viable tool to control catalysts conformation in enamine/iminium catalysis. ${ }^{[18]}$ However, with the introduction of metal fragments in the molecule, the catalysts described here cannot be considered, strictly speaking, an organocatalyst. Nevertheless it should be noted that the ferrocene moiety can act exclusively as a bulky group, in which the iron center is not involved in the reaction and does not provide attractive charge-dipole effects. These phenomena, particularly in $\mathrm{C}-\mathrm{F}$ bonds were recently used by Gilmour for 
an effective pre-organisation strategies for enantio- and diasteroselective reaction design in iminum catalysis. ${ }^{[19]}$

To start designing some possible structures, we considered the frame of the Hayashi-Jørgensen catalyst, and replaced the $\mathrm{OSiMe}_{3}$ group with a ferrocenyl group. In order to avoid the preparation of many derivatives, and the attempts to separate the enantiomers by chemical resolution, we started with a careful theoretical investigation, on this initial designed catalyst. This molecule possess two major conformational degrees of freedom (CDF) associated to the dihedral angles $\omega$, defined as $\mathrm{C} 5-(\mathrm{C} 1-\mathrm{C} 6)-\mathrm{C} 7$, and $\phi$, defined as $\mathrm{C} 1-$ (C6-C7)-N8 (Figure 2). To discover the conformation equili-
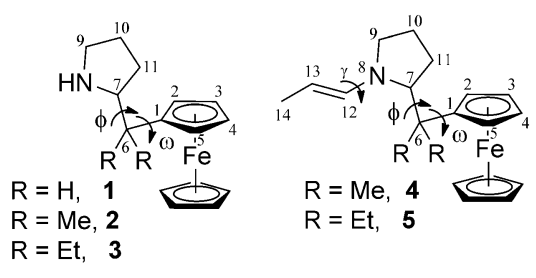

Figure 2. Geometrical parameters defining the conformational space of the ferrocenyl pyrrolidine catalyst.

brium of the investigated catalyst, we employed accurate quantum mechanical calculations (RIJCOSX-B3LYP/def2TZVPP optimization). Since the full exploration of the conformational space of the enamine adduct would have required an overwhelming effort, a different strategy was put in place. The enamine was constructed stepwise by adding molecular fragments to an initial ferrocene; at each step the relevant degrees of freedom were explored and the lowest conformer underwent the following step. Besides a noticeable saving in computing time, this approach provided useful insights on the effects of each molecular fragment to the overall conformational equilibrium. Both metal-carbon (2.09 A) and cyclopentadienyl carbon-carbon distances, obtained by optimization of eclipsed and staggered conformations of the unsubstituted ferrocene (model Fe $\mathbf{1}$ and Fe 2 in the Supporting Information), were in close agreement with those reported previously. ${ }^{[20]}$ The optimized staggered conformation $(\mathrm{Fe}-\mathrm{C}=2.081 \AA, \mathrm{C}-\mathrm{C}=1.422 \AA$ ) was used to build a tert-butyl derivative (model M1, see the Supporting Information) in order to study the energy difference between two possible rotamers M1 1 and 2 (Supporting Information). Geometry optimizations revealed M1 1 to be a stable minimum on the potential energy surface (PES); no other stable conformers were individuated. The substitution of a methyl with the pyrrolidine ring increases the number of possible conformers. Three stable rotamers were identified by relaxed scan along the $\phi$ dihedral angle, with the three minima lying in an energy range as narrow as $1.44 \mathrm{kcal}$ $\mathrm{mol}^{-1}$. Alkyl and aryl groups were then introduced to assess the influence exerted on this conformational equilibrium by C6-substituents of different size and shape. We expected to find a direct and linear correlation between the size of the substituents and their capability in controlling the conforma- tional equilibrium. Quite remarkably we noticed that the energy spread of conformers is significantly reduced by aryl groups, while much smaller and simpler alkyl chains were able to favor only one conformer (M3-S, see the Supporting Information) over the possible ones. In particular ethyl moieties showed the best compromise between effectiveness and size (Figure 3).

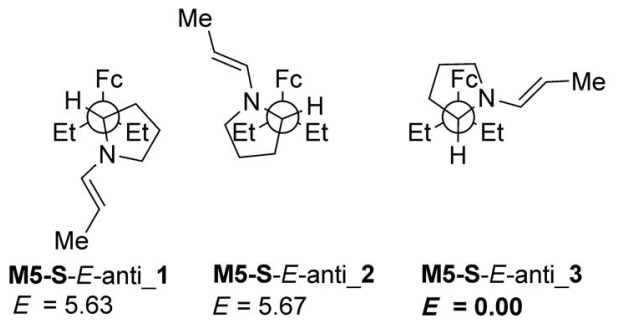

Figure 3. Preferred conformations of the enamine 5, calculated at the RIJCOSX-B3LYP/def2-TZVPP level. Energies in $\mathrm{kcal} \mathrm{mol}^{-1}$ (relative to the lowest energy structure).

The rotational equilibrium around the $\mathrm{C} 6-\mathrm{C} 7$ bond was investigated for both methyl- (M4-S, Supporting Information) and ethyl- (M5-S, Supporting Information) substituted enamine adducts. In the presence of methyl substituents the energy spread between rotamers is as small as in the pyrrolidine-substituted system (M2-S). The presence of an ethyl group strongly reduces the conformational freedom around this bond, as only one rotamer was predicted to be populated. In this rotamer, one of the ethyl groups is hindering the bottom face of the enamine $\mathbf{5}$.

Based on the theoretical analysis, we selected the diethyl ferrocenyl pyrrolidine $\mathbf{3}$ as a candidate for the synthetic evaluation. The synthesis of the enantiomerically pure $\mathbf{3}$ was devised as reported in Scheme 1.

The ferrocenyl ethyl ketone $\mathbf{6}^{[21]}$ was transformed in a straightforward manner in the tertiary alcohol. The resulting product was then treated with pyrrole in the presence of a catalytic amount of $\operatorname{InBr}_{3}(10 \mathrm{~mol} \%)$ to give 7 in high yield. $^{[22]}$ No traces of the $\beta$-pyrrole isomer was detected. Due to the reactivity of pyrrole, the reaction was conducted using an excess of this reagent at low temperature. The compound 7, after chromatographic purification, was hydrogenated with 1 atmosphere of hydrogen by using a rhodium on graphite ${ }^{[23]}$ catalyst to give the racemic pyrrolidine $\mathbf{3}$ in high yield. The racemic mixture was then separated by the synthesis of the corresponding $O$-acetyl-mandelic amide $\mathbf{8}^{[24]}$ The chromatographic separation by preparative TLC was quite straightforward. The hydrolysis of the isolated diasteroisomers of $\mathbf{8}$ proved to be quite difficult. In standard reaction conditions and in the presence of excess of $\mathrm{KOH}$ or $\mathrm{NaOH}$ no reaction was observed. However, by the treatment of the amide with an excess of $t \mathrm{BuOK}$ in THF under refluxing conditions ${ }^{[25]}$ for $36 \mathrm{~h}$, we were able to cleave the amide bond. The desired enantioenriched 2-(diethylferrocenyl)pyrrolidine $\mathbf{3}$ was isolate in satisfactory yields. 

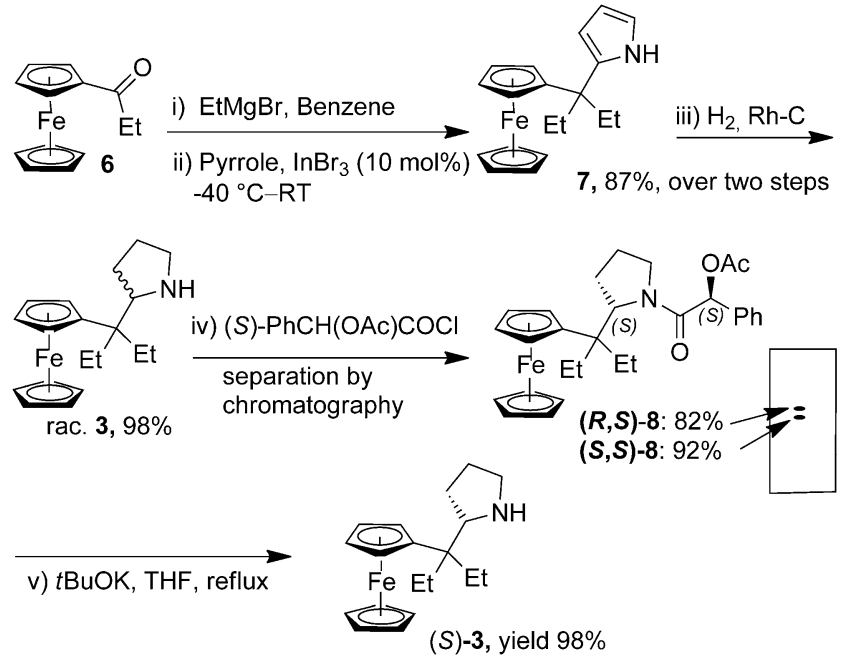

Scheme 1. Synthesis of racemic and enantioenriched ferrocenylpyrrolidine.

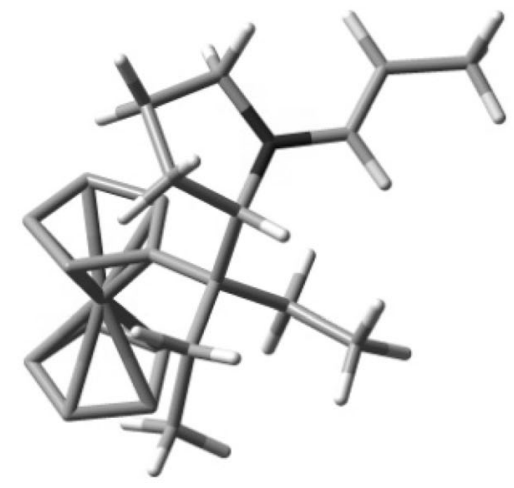

Calcd.

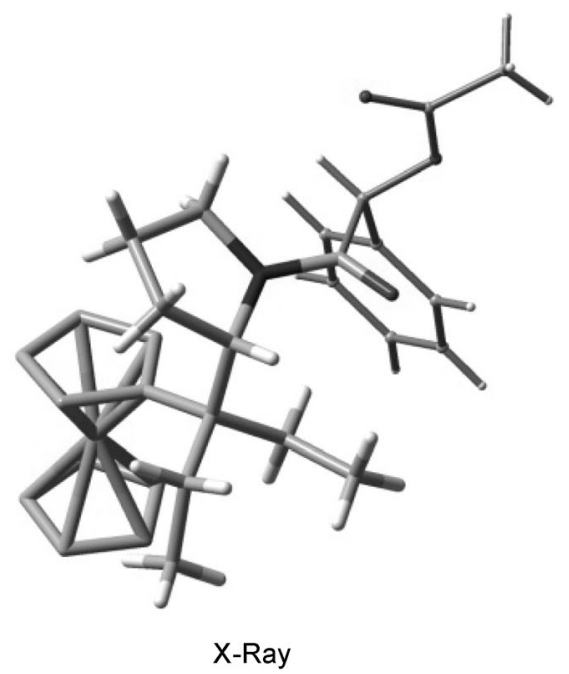

Figure 4. Determination of the configuration of the slow eluted amide obtained by reaction of $\mathbf{3}$ with $(S)$-OAc-mandelic chloride.

The absolute $(S, S)$ configuration of the more retained diastereoisomer was established by X-ray analysis (see the Supporting Information for further details). The X-ray structure of the mandelate was almost identical to the structure of the lowest energy conformer (Figure 4). The enamine 5 can be obtained in situ (in [D6]DMSO) by the direct reaction of propanal with the catalyst $3{ }^{[26]}$ A careful 1 D NOESY study, performed on the stable enamine, confirmed both the $E$ geometry of the enamine, and the conformation of the enamine calculated by DFT, thus ratifying the reliability of the theoretical studies. (See the Supporting Information for details). Next, $(S)$-2-(diethylferrocenyl)pyrrolidine 3 (96-99\% $e e$ as proven by its HPLC analysis after derivatization to the corresponding 3,5-dinitrophenylamide; see the Supporting Information for details and for HPLC traces), was applied in catalytic asymmetric nitro-Michael addition reactions of aldehydes $9 \mathbf{a}$ and $\mathbf{c}$ to nitro alkene $10 \mathbf{a}$ and $\mathbf{c}$ (Table 1).

Table 1. Organocatalytic addition of aldehydes to nitroalkenes promoted by 3. $^{[\mathrm{a}]}$

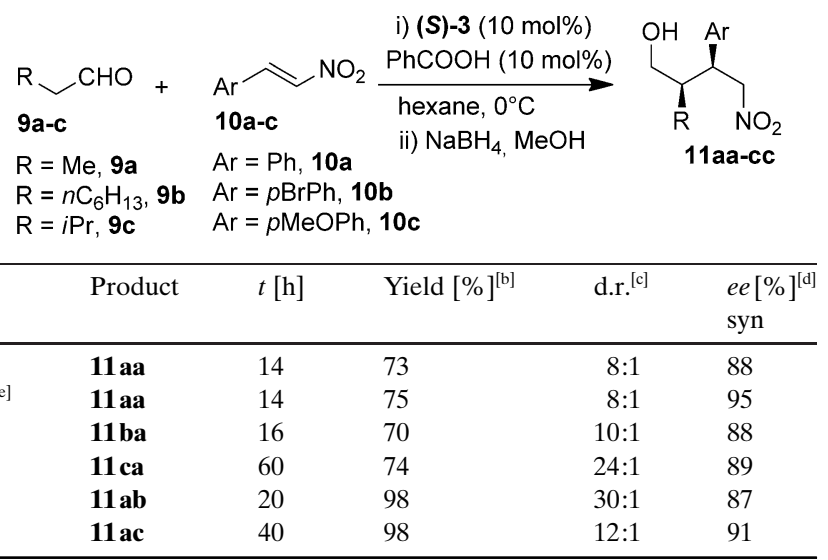

[a] The reactions were performed at $0^{\circ} \mathrm{C}$ with 1 equivalent of nitroalkene 10, 10 equivalents of aldehyde $\mathbf{9}$, in the presence of $10 \mathrm{~mol} \%$ of catalyst (S)-3 $(96 \% e e)$ and the reactions were run until completion checked by TLC $(16-60 \mathrm{~h})$. [b] Yield after chromatographic purification. [c] For all the reactions the d.r. (syn vs. anti) was measured by ${ }^{1} \mathrm{H}$ NMR and HPLC analysis. [d] Determined by chiral HPLC analysis. See Supporting Information for details. [e] $(S)-\mathbf{3}$ of $99 \%$ ee was used for the reaction.

Solvent screening experiments showed that hexane was the optimal solvent as it gives the best results in terms of stereoselectivity and yield. To our delight the excellent results, obtained under selected reaction conditions (see Table 1), confirmed our hypothesis about the ability of the catalyst in discriminating one face of the enamine.

It should be noted that, in contrast to the Hayashi-Jørgensen catalyst, the ferrocenyl compound $\mathbf{3}$ is a remarkably robust and recoverable catalyst and is not easily decomposed by acids or bases. In order to prove that the catalyst was of general use, we tested the performance of $\mathbf{3}$ in organocatalytic $\mathrm{S}_{\mathrm{N}}$ 1-type alkylations ${ }^{[27]}$ of enamines with the bis[4-(dimethylamino)phenyl] methanol $\mathbf{1 2}$ and the propargylic alcohol 14 (Scheme 2). Pleasantly, we found the catalyst gave satisfactory results in line with the recently developed silyl modified analogues of 2-tritylpyrrolidine, ${ }^{[12 b]}$ showing that the catalyst $\mathbf{3}$ was also able to promote different organocatalytic reactions. It worth mentioning that the 


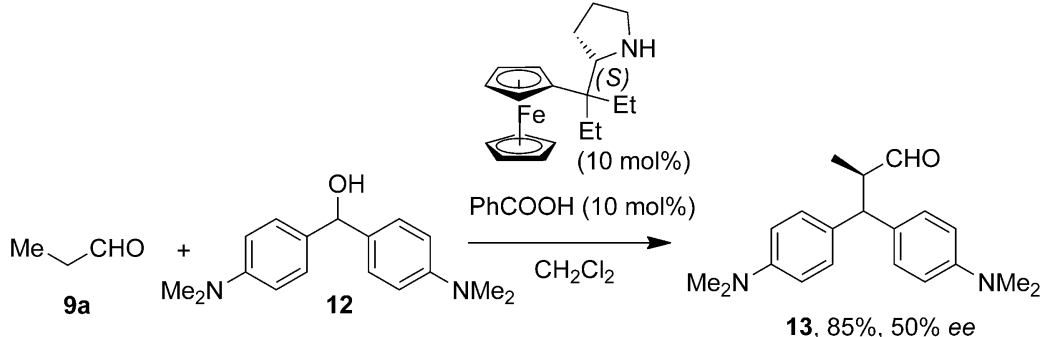

$13,85 \%, 50 \%$ ee

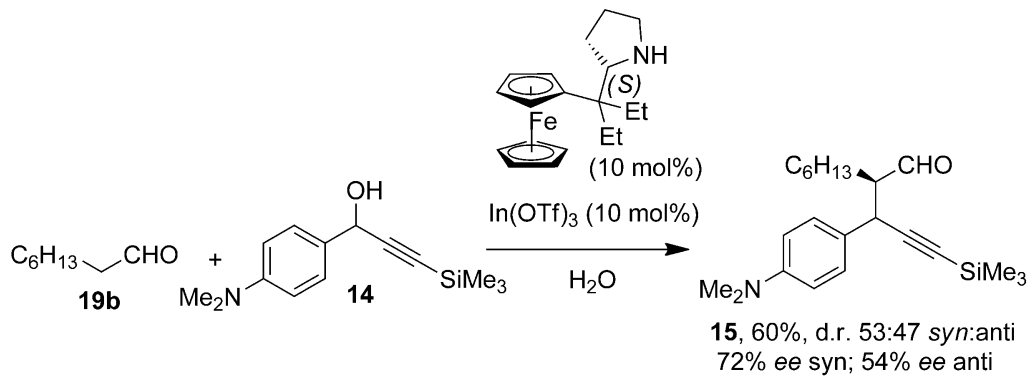

Scheme 2. $\mathrm{S}_{\mathrm{N}} 1$-type reaction of the alcohols $\mathbf{1 2}$ and $\mathbf{1 4}$ with the aldehydes $\mathbf{9 a}$ and $\mathbf{b}$ promoted by $10 \mathrm{~mol} \%$ of the catalyst 3 .

Hayashi-Jørgensen catalyst is not compatible with the presence of $\mathrm{In}^{\mathrm{III}}$ salts in the reaction mixture. ${ }^{[28]}$

We have performed DFT calculations in order to clarify the selectivity of the nitro Michael reaction (See the Supporting Information for full details). ${ }^{[29]}$ The conformational constraints imposed by ferrocene also drive the conformation of the ethyl groups, thus resulting in the effective screening of one face of the enamine intermediate. The less hindered enamine face reacts with the nitro derivative giving a zwiterrionic intermediate.

In conclusion, we have developed a new 2-(diethylferrocenyl) pyrrolidine catalyst, active in benchmark organocatalytic reactions. The ferrocenyl moiety, in combination with simple ethyl chains, is capable of fixing the enamine conformation addressing the approach trajectory of the nucleophile reaction partner. The results presented here represent an interesting proof-of-concept, showing for the first time the remarkable effectiveness of the ferrocenyl moiety in providing enantioselectivity through conformational selection. ${ }^{[30]}$ This approach could be viably employed in the rational design of ligands for organometallic or organocatalytic reactions.

\section{Experimental Section}

General procedure for the synthesis of compounds 12: In a vial the enantioenriched catalyst $(S)-3(3.3 \mathrm{mg}, 0.01 \mathrm{mmol}, 0.05$ equiv.) was dissolved in hexane, then the nitro alkene $(0.2 \mathrm{mmol}, 1$ equiv. $)$ and benzoic acid ( $1.2 \mathrm{mg}, 0.01 \mathrm{mmol}, 0.05$ equiv.) were added. The mixture was cooled to $0{ }^{\circ} \mathrm{C}$ and then the aldehyde $(2 \mathrm{mmol}, 10$ equiv. $)$ was added at $0^{\circ} \mathrm{C}$. The reaction mixture was stirred at $0 \mathrm{C}^{\circ}$ temperature for $10-24 \mathrm{~h}$. The reaction was quenched at $0^{\circ} \mathrm{C}$ by the addition of $\mathrm{HCl}(1 \mathrm{~mL}, 1 \mathrm{~N})$, then the mixture was extracted with diethyl ether $(2 \times 3 \mathrm{~mL})$. The organic phases were collected, dried over $\mathrm{Na}_{2} \mathrm{SO}_{4}$ and evaporated under reduced pressure. The crude product was analyzed with chiral HPLC: IA column; $n$ - hexane/isopropanol $\quad 95: 5 ; \quad$ flux $=$ $1.0 \mathrm{mLmin}^{-1} ; \quad T=30^{\circ} \mathrm{C}$. The crude product was then purified by flash chromatography on silica (cyclohexane/ethyl acetate) gives the pure products in 90 to $99 \%$ yield.

\section{Acknowledgements}

PRIN (Progetto Nazionale Stereoselezioni in Chimica Organica: Metodologie ed Applicazioni), Bologna University, Fondazione Del Monte and the European Commission through the project FP7-201431(CATAFLU.OR) are acknowledged for financial support

Keywords: enamines

ferrocene • nitro-Michael reaction $\cdot$ organocatalysis pyrrolidine

[1] For reviews, see: a) P. Melchiorre, M. Marigo, A. Carlone, G. Bartoli, Angew. Chem. 2008, 120, 6232-6265; Angew. Chem. Int. Ed. 2008, 47, 6138-6171; b) C. F. Barbas III, Angew. Chem. 2008, 120, 44-50; Angew. Chem. Int. Ed. 2008, 47, 42-47; c) G. Lelais, D. W. C. MacMillan, Aldrichimica Acta 2006, 39, 79-87.

[2] a) S. B. Jones, B. Simmons, D. W. C. MacMillan, J. Am. Chem. Soc. 2009, 131, 13606-13607; b) J. Carpenter, A. B. Northrup, D. M. Chung, J. J. M. Wieners, S.-G. Kim, D. W. C. MacMillan, Angew. Chem. 2008, 120, 3624-3628; Angew. Chem. Int. Ed. 2008, 47, $3568-$ 3572.

[3] J. E. Wilson, A. D. Casarez, D. W. C. MacMillan, J. Am. Chem. Soc. 2009, 131, 11332-11333.

[4] D. W. C. MacMillan, Nature 2008, 455, 304-308.

[5] A. Errkilä, I. Majander, P. M. Pihko, Chem. Rev. 2007, 107, 54165470.

[6] S. Mukherjee, J. W. Yang, S. Hoffmann, B. List, Chem. Rev. 2007, 107, 5471-5569.

[7] H. Mayr, S. Lakhdar, B. Maji, A. R. Ofial, Beilstein J. Org. Chem. 2012, 8, 1458-1478.

[8] D. Seebach, U. Grosělj, D. M. Badine, W. B. Schweizer, A. K. Beck, Helv. Chim. Acta 2008, 91, 1999-2034.

[9] For the first reports on Jørgensen-Hayashi catalysts, see: a) M. Marigo, T. C. Wabnitz, D. Fielenbach, K. A. Jørgensen, Angew. Chem. 2005, 117, 804-807; Angew. Chem. Int. Ed. 2005, 44, 794797; b) Y. Hayashi, H. Gotoh, T. Hayashi, M. Shoji, Angew. Chem. 2005, 117, 4284-4287; Angew. Chem. Int. Ed. 2005, 44, 4212-4215. For reviews see: C. Palomo, A. Mielgo, Angew. Chem. 2006, 118, 8042-8046; Angew. Chem. Int. Ed. 2006, 45, 7876-7880;K. L. Jensen, G. Dickmeiss, H. Jiang, Ł. Albrecht, K. A. Jørgensen, Acc. Chem. Res. 2012, 45, 248-264.

[10] C. Palomo, A. Landa, A. Mielgo, M. Oiarbide, A. Puente, S. Vera, Angew. Chem. 2007, 119, 8583-8587; Angew. Chem. Int. Ed. 2007, 46, 8431-8435.

[11] T. Kano, H. Mii, K. Maruoka, J. Am. Chem. Soc. 2009, 131, 34503451.

[12] a) R. Husmann, M. Jçrres, G. Raabe, C. Bolm, Chem. Eur. J. 2010, 16, 12549-12558; b) J. O. Bauer, J. Stiller, E. Marquéz López, K. Strohfeldt, M. Christman, C. Strohmann, Chem. Eur. J. 2010, 16, 
12553-12558; c) K. I. Jentzsch, T. Min, J. I. Etcheson, J. C. Fettinger, A. K. Franz, J. Org. Chem. 2011, 76, 7065-7075.

[13] a) R. Noyori, Angew. Chem. 2002, 114, 2108-2123; Angew. Chem. Int. Ed. 2002, 41, 2008-2022; b) B. M. Trost, J. Org. Chem. 2004, 69, 5813-5837.

[14] a) D. Seebach, A. K. Beck, M. Schiess, L. Widler, A. Wonnacott, Pure Appl. Chem. 1983, 55, 1807-1822; b) D. Seebach, A. K. Beck, R. Imwinkelried, S. Roggo, A. Wonnacott, Helv. Chim. Acta 1987, 70, 954-974; c) H. Pellissier, Tetrahedron 2008, 64, 10279-10317.

[15] T. Hintermann, D. Seebach, Helv. Chim. Acta 1998, 81, 2093-2126; C. Gaul, B. W. Schweizer, P. Seiler, D. Seebach, Helv. Chim. Acta 2002, 85, 1546-1566.

[16] R. Gómez Arrayás, J. Adrio, J. C. Carretero, Angew. Chem. 2006, 118, 7836-7878; Angew. Chem. Int. Ed. 2006, 45, 7674-7715.

[17] For a leading and important discussion about the "geminal-diaryl" effect for conformational fixation and stereodirection in these structures, see: M. Braun, Angew. Chem. 2012, 124, 2600-2612; Angew. Chem. Int. Ed. 2012, 51, 2550-2562; for the diaryl effect and its importance in organocatalysis, see: U. Grosĕlj, D. Seebach, D. M. Badine, W. B. Schweizer, A. K. Beck, I. Krossing, P. Klose, Y. Hayashi, T. Uchimaru, Helv. Chim. Acta 2009, 92, 1225-1259.

[18] For effective and powerful "organocatalysts" bearing a ferrocene moiety, see: G. Fu, Acc. Chem. Res. 2004, 37, 542-547.

[19] a) E.-M. Tanzer, L. E. Zimmer, W. B. Schweizer, R. Gilmour, Chem. Eur. J. 2012, 18, 11334-11342; b) L. E. Zimmer, C. Sparr, R. Gilmour, Angew. Chem. 2011, 123, 12062-12074; Angew. Chem. Int. Ed. 2011, 50, 11860-11871.

[20] S. Coriani, A. Halaand, T. Helgaker, P. Jørgensen, ChemPhysChem 2006, 7, 245-249.

[21] S. Top, B. Dauer, J. Vaissermann, G. Jaouen, J. Organomet. Chem. 1997, 541, 355-361.

[22] P. Vicennati, P. G. Cozzi, Eur. J. Org. Chem. 2007, 2248-2253.

[23] G. Falini, A. Gualandi, D. Savoia, Synthesis 2009, 2440-2446.

[24] M. Bandini, M. Gazzano, P. G. Cozzi, A. Umani-Ronchi, Eur. J. Org. Chem. 2001, 1937-1942.
[25] For a discussion about hydrolysis of amides, see: J. Aubé, Angew. Chem. 2012, 124, 3117-3119; Angew Chem. Int. Ed, 2012, 51, 3063 3065, and references therein.

[26] a) M. B. Schmid, K. Zeitler, R. M. Gschwind, J. Am. Chem. Soc. 2011, 133, 7065-7074; b) M. B. Schmid, K. Zeitler, R. M. Gschwind, Chem. Sci. 2011, 2, 1793-1803.

[27] For a highlight, see: P. G. Cozzi, F. Benfatti, Angew. Chem. 2010, 122, 264-267; Angew. Chem. Int. Ed. 2010, 49, 256-259. See: a) P. G. Cozzi, F. Benfatti, L. Zoli, Angew. Chem. 2009, 121, 1339 1342; Angew. Chem. Int. Ed. 2009, 48, 1313-1316; b) M. Guiteras Capdevila, F. Benfatti, L. Zoli, M. Stenta, P. G. Cozzi, Chem. Eur. J. 2010, 16, 11237-11241; c) A. Gualandi, E. Emer, M. Guiteras Capdevila, P. G. Cozzi, Angew. Chem. 2011, 123, 7988-7992; Angew. Chem. Int. Ed. 2011, 50, 7842-7846.

[28] a) K. Motoyama, M. Ikeda, Y. Miyake, Y. Nishibayashi, Eur. J. Org. Chem. 2011, 2239-2246; b) R. Sinisi, M. V. Vita, E. A. Gualandi, E. Emer, P. G. Cozzi, Chem. Eur. J. 2011, 17, 7404-7408.

[29] a) K. Patora-Komisarska, M. Benohoud, H. Ishikawa, D. Seebach Y. Hayashi, Helv. Chim. Acta 2011, 94, 719-745; b) J. Burès, A Armstrong, D. G. Blackmond, J. Am. Chem. Soc. 2011, 133, 88228823 ; c) G. Talavera, E. Reyes, J. L. Vicario, L. Carrillo, Angew. Chem. 2012, 124, 4180-4183; Angew. Chem. Int. Ed. 2012, 51, $4104-$ 4107; d) J. Duschmalé, J. Wiest, M. Wiesne, H. Wennemers, Chem. Science 2013, 4, 1312-1318; e) G. Sahoo, H. Rahaman, A. Madarász, I. Pápai, M. Melarto, A. Valkonen, P. M. Pihko, Angew. Chem. 2012 124, 13321-13325; Angew. Chem. Int. Ed. 2012, 51, 13144-13148; for a highlight, see: C. Moberg, Angew. Chem. 2013, 125, 2214 2216; Angew. Chem. Int. Ed. 2013, 52, 2160-2162.

[30] Recently, constrained pyrrolidine catalysts, in which the rigidity of the bicyclic structure imposes a synclinal endo disposition of the bulky substituents with respect to the pyrrolidine ring, were described, see: M. Lombardo, A. Quintavalla, E. Montroni, C. Trombini, Adv. Synth. Catal. 2012, 354, 3428-3443.

Received: March 13, 2013 Published online: $\mathbf{\square}$ u, 0000 


\section{Organocatalysis}

D. Petruzziello, M. Stenta,

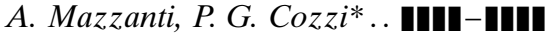

A Rational Approach Towards a New Ferrocenyl Pyrrolidine for Stereoselective Enamine Catalysis

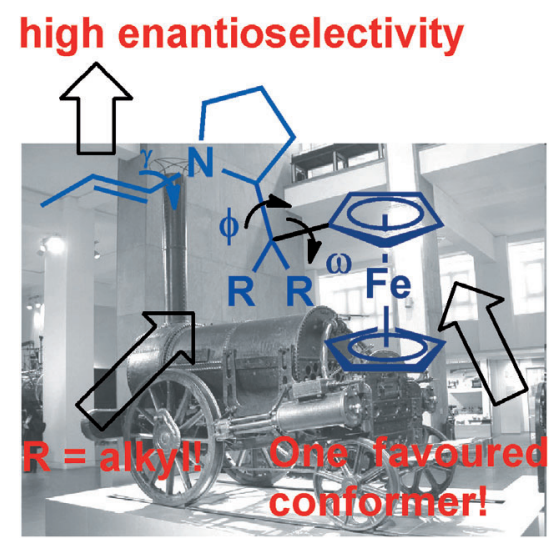

Ironing out the details: Proline and pyrrolidine derivatives (Hayashi-Jørgensen catalysts) are considered "work horses" in organocatalysis. This report describes a new effective ferrocenyl pyrrolidine catalyst able to perform well in benchmark organocatalytic reactions (see figure). The ferrocene moiety controls the conformational space and a simple alkyl group effectively covers a face of the derived enamine. This new framework can find applications in organocatalysis, and in general, in new ligand design. 